\title{
Bifurcation Analysis and Single Traveling Wave Solutions of the Variable-Coefficient Davey-Stewartson System
}

\author{
Tianyong Han $\mathbb{D D}^{1,2}$ Jiajin Wen, ${ }^{1}$ and $\mathrm{Zhao} \mathrm{Li} \mathbb{D}^{1}$ \\ ${ }^{1}$ College of Computer Science, Chengdu University, Chengdu 610106, China \\ ${ }^{2}$ Geomathematics Key Laboratory of Sichuan Province (Chengdu University of Technology), Chengdu 610059, China
}

Correspondence should be addressed to Tianyong Han; hantian123_123@163.com

Received 12 October 2021; Revised 13 December 2021; Accepted 28 December 2021; Published 13 January 2022

Academic Editor: Rigoberto Medina

Copyright (C) 2022 Tianyong Han et al. This is an open access article distributed under the Creative Commons Attribution License, which permits unrestricted use, distribution, and reproduction in any medium, provided the original work is properly cited.

This paper mainly studies the bifurcation and single traveling wave solutions of the variable-coefficient Davey-Stewartson system. By employing the traveling wave transformation, the variable-coefficient Davey-Stewartson system is reduced to two-dimensional nonlinear ordinary differential equations. On the one hand, we use the bifurcation theory of planar dynamical systems to draw the phase diagram of the variable-coefficient Davey-Stewartson system. On the other hand, we use the polynomial complete discriminant method to obtain the exact traveling wave solution of the variable-coefficient Davey-Stewartson system.

\section{Introduction}

Partial differential equations (PDEs) play a major role in the fields of plasma, quantum mechanics, and engineering [1]. In the study of PDEs, the most important thing is to analyze the dynamic behavior and find the exact traveling wave solution. In recent years, the study of exact traveling wave solutions of nonlinear PDEs with the variable coefficients has always been the focus of mathematicians and physicists, and many experts and scholars $[2,3]$ have proposed many methods to find PDEs with the variable coefficients, such as variable-coefficient extended mapping method [4], Hirota's bilinear method [5], Lax integrability [6], and dynamical system approach [7, 8].

One of the most important PDEs is the variable-coefficient Davey-Stewartson system. In this paper, we consider the variable-coefficient Davey-Stewartson system $[9,10]$ :

$$
\left\{\begin{array}{l}
i \frac{\partial u}{\partial t}+a_{1}(t) \frac{\partial^{2} u}{\partial x^{2}}+a_{2}(t) \frac{\partial^{2} u}{\partial y^{2}}-b_{1}(t)|u|^{2} u+b_{2}(t) u v=0 \\
s_{1} \frac{\partial^{2} v}{\partial x^{2}}-\frac{\partial^{2} v}{\partial y^{2}}=s_{2} \frac{\partial^{2}|u|^{2}}{\partial x^{2}}
\end{array}\right.
$$

where $u=u(t, x, y)$ is the complex wave envelope, $v=v(t, x, y)$ is the real forcing function, $a_{1}(t)$ and $a_{2}(t)$ are real functions with respect to time $t$, which stand for group velocity dispersion terms, $b_{1}(t)$ and $b_{2}(t)$ represent the quadratic nonlinearity and cubic nonlinear coefficient term, respectively, and $s_{1}$ and $s_{2}$ are constants. When the coefficients in equation (1) are constant, equation (1) is called the Davey-Stewartson system [11-15]. It is a very important nonlinear Schrödinger equation, which is usually used to describe the nonlinear wave packet of finite depth.

In [10], Wei and her collaborators investigated equation (1) by the Lie group method and obtained the periodic solutions and elliptic function solutions. In [9], El-Shiekh and Gaballah obtained the dark soliton solutions and bright soliton solutions of equation (1) by the modified sineGordon equation method. Although some exact solutions of equation (1) have been obtained in references $[9,10]$, the analysis of the dynamic behavior and the classification of traveling wave solutions of this kind of equation have not been reported. Therefore, in this paper, we will further study the above two problems.

The structure of this paper is as follows. In Section 2, we use the bifurcation theory of planar dynamical systems to draw the phase diagram of the variable-coefficient DaveyStewartson system. In Section 3, we construct the 
classification of all single traveling wave solutions of the variable-coefficient Davey-Stewartson system by the complete discrimination system. In Section 4, we give a summary.

\section{Bifurcation Analysis of System (1)}

Consider the traveling wave transformation as follows:

$$
\begin{aligned}
u(t, x, y) & =U(\xi) e^{i \eta}, \\
v(t, x, y) & =V(\xi), \\
\xi & =\mu_{1} x+\mu_{2} y-\lambda(t), \\
\eta & =k_{1} x+k_{2} y-\theta(t),
\end{aligned}
$$

where $\mu_{1}, \mu_{2}, k_{1}$, and $k_{2}$ are constants and $\lambda(t)$ and $\theta(t)$ are functions defined on $t$.

Substituting (2) into (1) and separating the real and imaginary parts, we obtain

$$
\begin{aligned}
\left(a_{1}(t) \mu_{1}^{2}+a_{2}(t) \mu_{2}^{2}\right) U(\xi)+\left(\theta^{\prime}(t)-a_{1}(t) k_{1}^{2}-a_{2}(t) k_{2}^{2}\right) U-b_{1}(t) U^{3}+b_{2}(t) U V & =0 \\
\left(\lambda^{\prime}(t)-2 k_{1} \mu_{1} a_{1}(t)-2 k_{2} \mu_{2} a_{2}(t)\right) U^{\prime} & =0 \\
\left(s_{1} \mu_{1}^{2}-\mu_{2}^{2}\right) V^{\prime \prime} & =\mu_{1}^{2} s_{2}\left(U^{2}(\xi)\right)^{\prime \prime}
\end{aligned}
$$

In order to eliminate the terms $U^{\prime}(\xi)$, we set $\lambda(t)=2 \int\left(\mu_{1} k_{1} a_{1}(t)+\mu_{2} k_{2} a_{2}(t)\right) \mathrm{d} t, \quad \theta(t)=\int\left(k_{1}^{2} a_{1}(t)+\right.$ $\left.k_{2}^{2} a_{2}(t)\right) \mathrm{d} t+\theta_{0}, \quad b_{1}(t)=b_{1}\left(\mu_{1}^{2} a_{1}(t)+\mu_{2}^{2} a_{2}(t)\right), \quad$ and $b_{2}(t)=b_{2}\left(\mu_{1}^{2} a_{1}(t)+\mu_{2}^{2} a_{2}(t)\right)$, where $b_{1}, b_{2}$, and $\theta_{0}$ are arbitrary constants. Then, equation (3) can be reduced to

$$
\begin{aligned}
U^{\prime \prime}(\xi)-b_{1} U^{3}(\xi)+b_{2} U(\xi) V(\xi) & =0, \\
\left(s_{1} \mu_{1}^{2}-\mu_{2}^{2}\right) V^{\prime \prime} & =\mu_{1}^{2} s_{2}\left(U^{2}(\xi)\right)^{\prime \prime} .
\end{aligned}
$$

Integrating the second equation of (4) twice with respect to $\xi$, we have

$$
V(\xi)=\frac{s_{2} \mu_{1}^{2}}{s_{1} \mu_{1}^{2}-\mu_{2}^{2}} U^{2}(\xi)+c_{1} \xi+c_{0}
$$

where $c_{0}$ and $c_{1}$ are integration constants. Substituting equation (5) into the first equation of (4), we obtain

$$
U^{\prime \prime}(\xi)+\left(\frac{s_{2} \mu_{1}^{2} b_{2}}{s_{1} \mu_{1}^{2}-\mu_{2}^{2}}-b_{1}\right) U^{3}(\xi)+b_{2} c_{0} U(\xi)=0
$$

Next, by assuming $(\mathrm{d} U / \mathrm{d} \xi)=\psi$, we can rewrite equation (6) as the following planar dynamical system:

$$
\left\{\begin{array}{l}
\frac{\mathrm{d} U}{\mathrm{~d} \xi}=\psi \\
\frac{\mathrm{d} \psi}{\mathrm{d} \xi}=-\left(\frac{s_{2} \mu_{1}^{2} b_{2}}{s_{1} \mu_{1}^{2}-\mu_{2}^{2}}-b_{1}\right) U^{3}(\xi)-b_{2} c_{0} U(\xi),
\end{array}\right.
$$

with the Hamiltonian system

$H(U, \psi)=\frac{1}{2} \psi^{2}+\frac{1}{4}\left(\frac{s_{2} \mu_{1}^{2} b_{2}}{s_{1} \mu_{1}^{2}-\mu_{2}^{2}}-b_{1}\right) U^{4}(\xi)+\frac{b_{2} c_{0}}{2} U^{2}(\xi)=h$.
Here, we assume that $G(U)=-\left(\left(s_{2} \mu_{1}^{2} b_{2} /\left(s_{1} \mu_{1}^{2}-\right.\right.\right.$ $\left.\left.\left.\mu_{2}^{2}\right)\right)-b_{1}\right) U^{3}(\xi)-b_{2} c_{0} U(\xi)$. When $\quad\left(s_{2} \mu_{1}^{2} b_{2} /\right.$ $\left.\left.\left(s_{1} \mu_{1}^{2}-\mu_{2}^{2}\right)\right)-b_{1}\right) b_{2} c_{0}<0$, we easily obtain three zeros of $G(U) \quad$ including $\quad U_{0}=0, \quad U_{1}=$ $\sqrt{-\left(b_{2} c_{0}\left(s_{1} \mu_{1}^{2}-\mu_{2}^{2}\right) /\left(s_{2} \mu_{1}^{2} b_{2}-b_{1}\left(\left(s_{1} \mu_{1}^{2}-\mu_{2}^{2}\right)\right)\right)\right.}$, and $U_{2}=-$ $\sqrt{-\left(b_{2} c_{0}\left(s_{1} \mu_{1}^{2}-\mu_{2}^{2}\right) /\left(s_{2} \mu_{1}^{2} b_{2}-b_{1}\left(s_{1} \mu_{1}^{2}-\mu_{2}^{2}\right)\right)\right)}$. When $\left(\left(s_{2} \mu_{1}^{2} b_{2} / s_{1} \mu_{1}^{2}-\mu_{2}^{2}\right)-b_{1}\right) b_{2} c_{0}>0$, we obtain $U_{3}=0$. Then, we suppose that $M_{i}\left(U_{i}, 0\right)$ are the equilibrium points of equation (8), and we obtain that $M_{i}\left(U_{i}, 0\right)$ is the saddle point when $G^{\prime}\left(U_{i}\right)>0 ; M_{i}\left(U_{i}, 0\right)$ is the degraded saddle point when $G^{\prime}\left(U_{i}\right)=0 ; M_{i}\left(U_{i}, 0\right)$ is the center point when $G^{\prime}\left(U_{i}\right)<0$. With the help of Maple software, we draw the phase portraits of (8) as shown in Figure 1.

\section{Traveling Wave Solutions of System (1)}

The complete discriminant system method was first introduced by $\mathrm{Lu}$ and his collaborators in 1996 [16]. In recent years, many experts and scholars [17-22] have applied this method to construct the exact traveling wave solutions of partial differential equations. In this section, we intend to use this method to analyze the exact traveling wave solution of the variable-coefficient DaveyStewartson system.

In fact, in Section 2, we have simplified equation (1) to nonlinear equation (6). Next, multiply both ends of equation (6) by $U^{\prime}$ and integrate once, and we obtain

$$
\left(U^{\prime}\right)^{2}=d_{4} U^{4}(\xi)+d_{2} U^{2}(\xi)+d_{0}
$$

where $d_{4}=-1 / 2\left(\left(s_{2} \mu_{1}^{2} b_{2} / s_{1} \mu_{1}^{2}-\mu_{2}^{2}\right)-b_{1}\right), d_{2}=-b_{2} c_{0}$, and $d_{0}$ is the integration constant.

Consider the following transformations: 


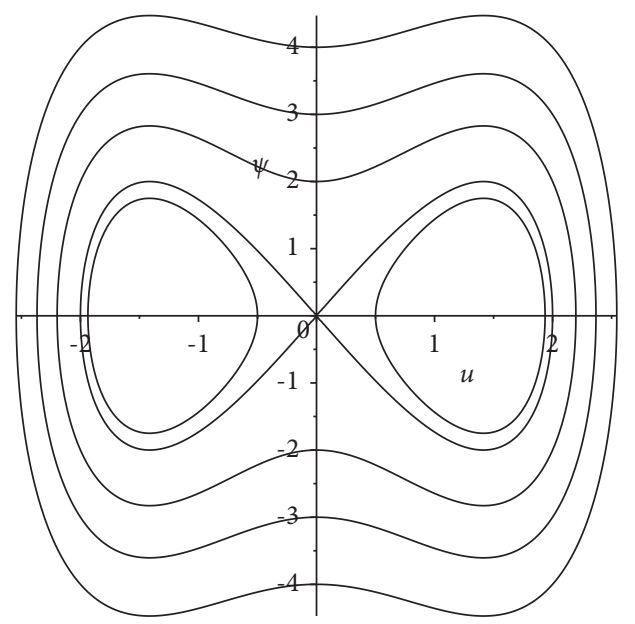

(a)

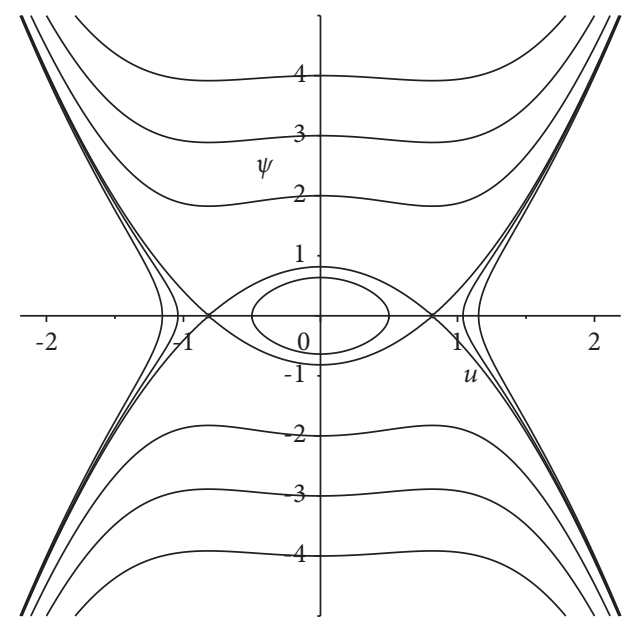

(b)

FiguRE 1: Phase portraits of system (7). (a) $\left(s_{2} \mu_{1}^{2} b_{2} / s_{1} \mu_{1}^{2}-\mu_{2}^{2}\right)-b_{1}>0,\left(b_{2} c_{0} / 2\right)<0$. (b) $\left(s_{2} \mu_{1}^{2} b_{2} / s_{1} \mu_{1}^{2}-\mu_{2}^{2}\right)-b_{1}<0,\left(b_{2} c_{0} / 2\right)>0$.

$$
\left\{\begin{array}{l}
U= \pm \sqrt{\left(4 d_{4}\right)^{-(1 / 3)} w} \\
p=4 d_{2}\left(4 d_{4}\right)^{-(2 / 3)} \\
q=4 d_{0}\left(4 d_{4}\right)^{-(1 / 3)} \\
\xi_{1}=\left(4 d_{4}\right)^{(1 / 3)} \xi
\end{array}\right.
$$

Then, equation (9) can be rewritten as

$$
\left(w_{\xi_{1}}\right)^{2}=w\left(w^{2}+p w+q\right)
$$

Integrating equation (11) once, we obtain

$$
\pm\left(\xi_{1}-\xi_{0}\right)=\int \frac{\mathrm{d} w}{\sqrt{w\left(w^{2}+p w+q\right)}}
$$

where $\xi_{0}$ is an integration constant. Setting $F(w)=w^{2}+p w+q$, its complete discrimination system is

$$
\Delta=p^{2}-4 q .
$$

According to the root of equation (13), the traveling wave solution of equation (1) has four cases.

Case 1. Assume that $\Delta=0$. Since $w>0$, we can obtain

$$
\pm\left(\xi_{1}-\xi_{0}\right)=\int \frac{\mathrm{d} w}{\sqrt{w}(w+(p / 2))} .
$$

If $p<0$, the explicit solution of equation (1) is

$$
\begin{aligned}
& u_{1}(t, x, y)= \pm \sqrt{-\frac{1}{2} d_{2} d_{4}^{-1} \tanh ^{2}\left[\frac{1}{2}\left(-2 d_{2}\left(4 d_{4}\right)^{-(2 / 3)}\right)^{(1 / 2)}\left(\left(4 d_{4}\right)^{(1 / 3)} \xi-\xi_{0}\right)\right]} e^{i\left(k_{1} x+k_{2} y-\theta(t)\right)} \\
& u_{2}(t, x, y)= \pm \sqrt{-\frac{1}{2} d_{2} d_{4}^{-1} \operatorname{coth}^{2}\left[\frac{1}{2}\left(-2 d_{2}\left(4 d_{4}\right)^{-(2 / 3)}\right)^{(1 / 2)}\left(\left(4 d_{4}\right)^{(1 / 3)} \xi-\xi_{0}\right)\right]} e^{i\left(k_{1} x+k_{2} y-\theta(t)\right)}
\end{aligned}
$$

If $p>0$, the explicit solution of equation (1) is

$$
u_{3}(t, x, y)=\sqrt{\frac{1}{2} d_{2} d_{4}^{-1} \tan ^{2}\left[\frac{1}{2}\left(2 d_{2}\left(4 d_{4}\right)^{-(2 / 3)}\right)^{(1 / 2)}\left(\left(4 d_{4}\right)^{(1 / 3)} \xi-\xi_{0}\right)\right]} e^{i\left(k_{1} x+k_{2} y-\theta(t)\right)}
$$

If $p=0$, the explicit solution of equation (1) is $u_{4}(t, x, y)=2 \sqrt{\frac{\left(4 d_{4}\right)^{-(1 / 3)}}{\left(\left(4 d_{4}\right)^{(1 / 3)} \xi-\xi_{0}\right)^{2}}} e^{i\left(k_{1} x+k_{2} y-\theta(t)\right)}$.
Case 2. Assume that $\Delta>0$ and $q=0$. Since $w>-p$, we can obtain

$$
\pm\left(\xi_{1}-\xi_{0}\right)=\int \frac{\mathrm{d} w}{w \sqrt{w+p}}
$$


According to Case 1, the solution of equation (18) is as If $p>0$, the explicit solution of equation (1) is follows.

$$
\begin{aligned}
& u_{5}(t, x, y)= \pm \sqrt{\frac{1}{2} d_{2} d_{4}^{-1} \tanh ^{2}\left[\frac{1}{2}\left(2 d_{2}\left(4 d_{4}\right)^{-(1 / 3)}\right)^{(1 / 2)}\left(\left(4 d_{4}\right)^{(1 / 3)} \xi-\xi_{0}\right)\right]-d_{2} d_{4}^{-1}} e^{i\left(k_{1} x+k_{2} y-\theta(t)\right)} \\
& u_{6}(t, x, y)= \pm \sqrt{\frac{1}{2} d_{2} d_{4}^{-1} \operatorname{coth}^{2}\left[\frac{1}{2}\left(2 d_{2}\left(4 d_{4}\right)^{-(1 / 3)}\right)^{(1 / 2)}\left(\left(4 d_{4}\right)^{(1 / 3)} \xi-\xi_{0}\right)\right]-d_{2} d_{4}^{-1}} e^{i\left(k_{1} x+k_{2} y-\theta(t)\right)} .
\end{aligned}
$$

If $p<0$, the explicit solution of equation (1) is

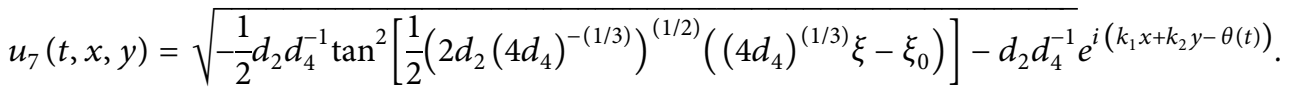

Case 3. Assume that $\Delta>0, q \neq 0$, and $\alpha<\beta<\gamma$, where one of $\alpha, \beta$, and $\gamma$ is zero, and the rest of them are the two roots of $F(w)$. Since $\alpha<w<\beta$, make the transformation $w=\alpha+(\beta-\alpha) \sin ^{2} \varphi$. It can be obtained from equation (13) that

$$
\pm\left(\xi_{1}-\xi_{0}\right)=\frac{2}{\sqrt{\gamma-\alpha}} \int \frac{\mathrm{d} \varphi}{\sqrt{1-m^{2} \sin ^{2} \varphi}}
$$

$$
u_{8}(t, x, y)=\sqrt{\left(4 d_{4}\right)^{-(1 / 3)}\left[\alpha+(\beta-\alpha) \mathbf{s n}^{2}\left(\frac{\sqrt{\gamma-\alpha}}{2}\left(\left(4 d_{4}\right)^{(1 / 3)} \xi-\xi_{0}\right), m\right)\right]} e^{i\left(k_{1} x+k_{2} y-\theta(t)\right)} .
$$

Make the transformation $w=\left(\left(-\beta \sin ^{2} \varphi+\gamma\right) / \cos ^{2} \varphi\right)$ and substitute it into equation (12). Similarly, the explicit solution of equation (1) can be obtained:

$$
u_{9}(t, x, y)=\sqrt{\left(4 d_{4}\right)^{-(1 / 3)} \frac{-\beta \mathbf{s n}^{2}\left((\sqrt{\gamma-\alpha} / 2)\left(\left(4 d_{4}\right)^{(1 / 3)} \xi-\xi_{0}\right), m\right)+\gamma}{\mathbf{c n}^{2}\left((\sqrt{\gamma-\alpha} / 2)\left(\left(4 d_{4}\right)^{(1 / 3)} \xi-\xi_{0}\right), m\right)}} e^{i\left(k_{1} x+k_{2} y-\theta(t)\right)}
$$

Case 4. Assume that $\Delta<0$. Since $w>0$, we can make the following transformation:

$$
w=\sqrt{q} \tan ^{2} \frac{\varphi}{2}
$$

Substituting equation (24) into equation (13), we obtain

$$
\pm\left(\xi_{1}-\xi_{0}\right)=q^{-(1 / 4)} \int \frac{\mathrm{d} \varphi}{\sqrt{1-k^{2} \sin ^{2} \varphi}}
$$

where $m^{2}=(1 / 2)(1-(p / 2 \sqrt{q}))$.
According to equation (25) and the definition of Jacobian elliptic function $\mathbf{c n}$, we obtain

$$
\mathbf{c n}\left((q)^{(1 / 4)}\left(\xi_{1}-\xi_{0}\right), m\right)=\cos \varphi .
$$

From equation (24), we have

$$
\cos \varphi=\frac{2 \sqrt{q}}{w+\sqrt{q}}-1
$$

Comparing equation (26) with equation (27), we can obtain the explicit solution of equation (1): 


$$
u_{10}(t, x, y)=\sqrt{\left(4 d_{4}\right)^{-(1 / 3)}\left[\frac{2 \sqrt{4 d_{0}\left(4 d_{4}\right)^{-(1 / 3)}}}{1+\mathbf{c n}\left(\left(4 d_{0}\left(4 d_{4}\right)^{-(1 / 3)}\right)^{(1 / 4)}\left(\left(4 d_{4}\right)^{(1 / 3)} \xi-\xi_{0}\right), m\right)}-\sqrt{4 d_{0}\left(4 d_{4}\right)^{-(1 / 3)}}\right]} e^{i\left(k_{1} x+k_{2} y-\theta(t)\right)} .
$$

Remark 1. In this paper, we obtained one of the solutions $(u(t, x, y))$ of equation (1). Using relation (5), we can obtain another solution $v(t, x, y)$ of equation (1).

\section{Conclusion}

In this paper, the bifurcation and single traveling wave solutions of the variable-coefficient Davey-Stewartson system have been investigated by employing the bifurcation theory of planar dynamical systems and the polynomial complete discriminant method. The phase portraits of the variable-coefficient Davey-Stewartson system are shown in Figure 1. Moreover, a series of new single traveling wave solutions is obtained. Compared with the published literature [9], this study not only obtains the hyperbolic function solution, trigonometric function solution, and rational function solution but also obtains the Jacobi function solution. We believe that the study of the variable-coefficient Davey-Stewartson system in the paper will help mathematicians and physicists.

\section{Data Availability}

No data were used to support this study.

\section{Conflicts of Interest}

The authors declare that they have no conflicts of interest.

\section{Acknowledgments}

This study was supported by Opening Fund of Geomathematics Key Laboratory of Sichuan Province (no. scsxdz2021yb05).

\section{References}

[1] L. Tang and S. Chen, "Traveling wave solutions for the diffusice Lotka-Volterra equations with boundary problems," Applied Mathematics and Computation, vol. 413, Article ID 126599, 2022.

[2] S. Kumar, D. Jyoti, K. S. Nisar, and M. Zakarya, "New exact static solutions of Einstein-Maxwell field equations with a magnetic dipole," Results in Physics, vol. 24, Article ID 104136, 2021.

[3] L. Tang, "Dynamical behavior and traveling wave solutions in optical fibers with Schrödinger-Hirota equation," Optik, vol. 245, Article ID 167750, 2021.

[4] S. Zhang and T. Xia, "Variable-coefficient extended mapping method for nonlinear evolution equations," Physics Letters A, vol. 372, no. 11, pp. 1741-1749, 2008.

[5] S. Zhang and X. Gao, "Exact N-soliton solutions and dynamics of a new AKNS equation with time-dependent coefficients," Nonlinear Dynamics, vol. 83, no. 1-2, pp. 1043-1052, 2016.
[6] S. Zhang and S. Y. Hong, "Lax integrability and exact solutions of a variable-coefficient and nonisospectral AKNS hierarchy," International Journal of Nonlinear Sciences and Numerical Simulation, vol. 19, pp. 251-262, 2016.

[7] Z.-Y. Zhang, Z.-H. Liu, X.-J. Miao, and Y.-Z. Chen, "Qualitative analysis and traveling wave solutions for the perturbed nonlinear Schrodinger's equation with Kerr law nonlinearity," Physics Letters A, vol. 375, no. 10, pp. 1275-1280, 2011.

[8] Z.-Y. Zhang, X.-Y. Gan, and D.-M. Yu, "Bifurcation behaviour of the travelling wave solutions of the perturbed nonlinear Schrodinger's equation with Kerr law nonlinearity," Zeitschrift für Naturforschung A, vol. 66, no. 12, pp. 721-727, 2014.

[9] R. M. Ei-Shiekh and M. Gaballah, "Solitary wave solutions for the variable-coefficient coupled nonlinear Schrödinger equations and Davey-Stewartson system using modified sineGordon equation method," Journal of Ocean Engineering and Science, vol. 5, pp. 180-185, 2020.

[10] G.-M. Wei, Y.-L. Lu, Y.-Q. Xie, and W.-X. Zheng, "Lie symmetry analysis and conservation law of variable-coefficient Davey-Stewartson equation," Computers \& Mathematics with Applications, vol. 75, no. 9, pp. 3420-3430, 2018.

[11] C. Qian, J. Rao, D. Mihalache, and J. He, "Rational and semirational solutions of they-nonlocal Davey-Stewartson I equation," Computers \& Mathematics with Applications, vol. 75, no. 9, pp. 3317-3330, 2018.

[12] L. Kavitha, B. Srividya, and D. Gopi, "Exact propagating dromion-like localized wave solutions of generalized $(2+1)$-dimensional Davey-Stewartson equations," Computers \& Mathematics with Applications, vol. 62, no. 12, pp. 4691-4707, 2011.

[13] S. Arshed, N. Raza, and M. Alansari, "Soliton solutions of the generalized Davey-Stewartson equation with full nonlinearities via three integrating schemes," Ain Shams Engineering Journal, vol. 12, no. 3, pp. 3091-3098, 2021.

[14] N. Taghizadeh, A. Neirameh, and S. Shokooh, "New exact solutions for Davey-Stewartson system," Journal of the Association of Arab Universities for Basic and Applied Sciences, vol. 12, no. 1, pp. 61-64, 2012.

[15] K. Boateng, W. Yang, W. O. Apeanti, and D. Yaro, "New exact solutions and modulation instability for the nonlinear $(2+1)$-dimensional Davey-Stewartson system of equation," Advances in Mathematical Physics, vol. 2019, Article ID 3879259, 15 pages, 2019.

[16] Y. Lu, X. Hou, and Z. Zeng, "A compete discrimation system for polynomial," Science in China, vol. 26, no. 6, pp. 628-646, 1996.

[17] Y. Xie, Z. Yang, and L. Li, "New exact solutions to the high dispersive cubic-quintic nonlinear Schrödinger equation," Physics Letters A, vol. 382, no. 36, pp. 2506-2514, 2018.

[18] C. Li, G. Li, and L. Chen, "Fractional optical solitons of the space-time perturbed fractional Gerdjikov-Ivanov equation," Optik, vol. 224, Article ID 165638, 2020.

[19] Y. Xie, L. Li, and Y. Kang, "New solitons and conditional stability to the high dispersive nonlinear Schrödinger equation with parabolic law nonlinearity," Nonlinear Dynamics, vol. 103, no. 1, pp. 1011-1021, 2021. 
[20] Z. Li, P. Li, and T. Han, "Dynamical behavior and the classification of single traveling wave solutions for the coupled nonlinear Schrödinger equation with variable coefficients," Advances in Mathematical Physics, vol. 2021, Article ID 9955023, 10 pages, 2021.

[21] L. Li, Y. Xie, and S. Zhu, "New exact solutions for a generalized KdV equation," Nonlinear Dynamics, vol. 92, no. 2, pp. 215-219, 2018.

[22] Z. Li and T. Han, "Classification of all single traveling wave solutions of fractional perturbed Gerdjikov-Ivanov equation," Mathematical Problems in Engineering, vol. 2021, Article ID 1283083, 7 pages, 2021. 